\title{
L'homoparentalité en questions
}

\author{
Chantal ZAOUCHE-GAUDRON, Olivier VECHO
}

Laboratoire Personnalisation et Changements Sociaux. Université Toulouse II le Mirail, Toulouse

\begin{abstract}
RESUME
L'homoparentalité suscite de multiples réflexions souvent passionnées dans la mesure où elle fait partie dorénavant de notre paysage social. L'objectif de cette contribution est une mise en débat de l'homoparentalité. Après un bref rappel socio-historique qui a pour visée de mettre en exergue les changements de paradigmes en psychologie et une mise en perspective des données issues de l'anthropologie, nous examinerons les principales interrogations liées à l'apparition des familles homoparentales. Plusieurs éléments font nœuds dans le débat sur l'homoparentalité : la scène primitive et la question des origines, le désir d'enfant, la parenté biologique versus la parenté sociale. Le développement des enfants qui grandissent dans des familles homoparentales sera examiné à partir des résultats issus de la littérature scientifique. Ces différents points seront abordés et discutés.
\end{abstract}

Mots-clés : homoparentalité, scène primitive, secret des origines, développement des enfants

\section{INTRODUCTION}

Si toute recherche est historiquement datée, de façon parallèle, la connaissance scientifique entraîne des bouleversements sociaux, y compris là où on ne l'attendait pas. Ce faisant, le savoir médical, les progrès de la science avec les Procréations Médicalement Assistées (P.M.A.), les Fécondations In Vitro (F.I.V.), les Inséminations Avec Donneur (I.A.D.)... participent à l'avènement des parentalités singulières. Nous débuterons donc par une mise en perspective des mouvements socio-historiques des trois dernières décennies qui ont modifié la place, le statut, le rôle de chaque membre du système familial, et des paradigmes étudiés qui s'articulent avec eux. Les changements de paradigmes en psychologie sont, en effet, étroitement liés aux transformations sociales et juridiques qui concernent la famille.

Quel que soit leur champ disciplinaire, les auteurs (sociologue, historien, anthropologue, psychologue...) s'accordent à dire que les années 70 représentent un tournant historique majeur explicatif de l'évolution du système familial. Durant cette période, le mouvement féministe promeut l'égalité des rôles parentaux et l'égalité des hommes et des femmes au sein de l'espace domestique et de la sphère professionnelle. La liberté sexuelle et la maîtrise de la procréation sont autant de maîtres mots qui accompagnent ce mouvement ; de façon concomitante, sont promulguées les Lois qui abolissent la puissance paternelle et l'autorité paternelle. Dans le même temps, de nombreuses études mettent en évidence les compétences précoces de l'enfant faisant de lui une personne à part entière, capable d'interagir dès sa naissance (voire in utero) avec autrui. A partir des années 70 dans les pays Anglo-saxons et dans les années 80 en France, les recherches posent dorénavant des paradigmes comparatifs des relations mère-enfant versus père-enfant, délaissant le primat de la relation maternelle des psychanalystes et des théoriciens de l'attachement. De nombreux travaux relatent l'importance du rôle du père comparé à celui de la mère dans le développement affectif, cognitif, sexué de l'enfant, ou dans les relations d'attachement. Plusieurs études en sociologie et en psychologie s'attachent aussi à examiner la répartition des tâches des pères et des mères au sein du foyer et mettent en avant les fameux " nouveaux pères " présents dans les soins et l'éducation du jeune enfant dès l'aube de sa vie.

\section{Correspondance :}

Pr Chantal ZAOUCHE-GAUDRON - Laboratoire

Personnalisation et Changements Sociaux, Université Toulouse II le Mirail, 5 allées A. Machado, 31058 Toulouse Cedex 9 - Tel 0033 (0)542504825 -

Email zaouche@univ-tise2.fr 
Au cours des années 90, autre tournant juridique majeur dans notre société, l'exercice conjoint de l'autorité parentale après la séparation conjugale se légalise (Lois de 1987 et 1993). Durant cette période, émerge un champ de recherche en psychologie qui examine plus particulièrement la relation père-enfant : on parle alors de différenciation paternelle [42], d'ímplication [27], de sensibilité, de disponibilité, de proximité paternelle [26], de relation d'attachement pèreenfant [34]. Ce paradigme s'appuie sur la qualité et la spécificité de la relation père-enfant à l'œuvre dès la prime enfance, et instaure un débat entre la psychologie du développement, la psychanalyse et la sociologie [43].

Enfin, au cours de l'année 2002, la Loi du 4 mars instaure la résidence alternée après les séparations conjugales, et offre aussi la possibilité pour les parents d'obtenir le droit de demander " lorsque les circonstances l'exigent » le partage de l'autorité parentale avec " un tiers de confiance ». C'est ainsi que des couples de gays et de lesbiennes obtiennent parfois - quand ils en font la demande - la délégation de l'autorité parentale. Les recherches sur le couple conjugal, le couple parental et la co-parentalité ne cessent de croître en raison de la modification du paysage social dans lequel on relève, d'une part, une augmentation des séparations conjugales, et d'autre part, une diversification des structures familiales (monoparentales, recomposées, familles homoparentales, situations familiales avec résidence alternée...).

Plus précisément en France, naissent en 1986 l'Association des Parents et futurs parents Gays et Lesbiens (A.P.G.L.) [2], et l'adoption, en 1997, d'un nouveau vocable " l'homoparentalité "; la famille homoparentale s'inscrit dès lors dans notre réalité sociale. "La notion de famille homoparentale désigne un ensemble de personnes constitué de deux groupes : une structure parentale formée d'un parent unique ou d'un couple de parents dont l'orientation homosexuelle est individuellement claire et collectivement reconnue ; d'autre part, un ou plusieurs enfants légalement considérés comme issus d'un des parents au moins » [29]. Au risque de simplifier la complexité de la réalité historique, relevons ici la succession de deux temps : au cours du premier, la sexualité qui se délie de la procréation (via les méthodes contraceptives) entraîne, entre autres, la reconnaissance sociale de l'homosexualité ; dans le second temps, la conjugalité qui se disjoint peu à peu de la parentalité suscite l'identification et l'étude des parentés plurielles y compris celles de l'homoparentalité. En 2004, le Tribunal de Grande Instance de Paris reconnaît la première famille homoparentale en France. Les enfants portent le nom de la femme qui les a conçus par I.A.D., et la conjointe obtient la possibilité d'adopter les enfants.

\section{REGARDS ANTHROPOLOGIQUES}

Pour Godelier [23] « dans l'immense majorité des sociétés connues, les rapports de parenté naissent de la mise en pratique des principes communément admis par les individus ". Les principes invoqués relèvent du mariage, de la filiation, de la prohibition de l'inceste et des rapports de soli- darité entre les sexes et les générations. Godelier [18] relate que dans certaines sociétés existent des couples de même sexe qui élèvent des enfants mais qui n'ont pas de rapports sexuels entre eux. Ainsi, chez les Nuers du Soudan, une femme stérile ou ménopausée peut se marier avec une femme plus jeune sans partager sa couche. La jeune femme peut avoir des amants et, à la naissance d'un enfant, la femme plus âgée devient alors le père du nourrisson. II y a ici homoparentalité sans rapports homosexuels. A contrario, chez les Azandés, pour faire face à la pénurie de femmes due à la polygamie des hommes âgés, les jeunes hommes contractent des unions homosexuelles temporaires mais sans adoption d'enfants, avant de se marier dans un second temps avec la sœur de leur conjoint. Ici, il y a homosexualité sans homoparentalité. Ainsi dans les deux cas présentés, l'homosexualité et la parentalité apparaissent disjointes l'une de l'autre.

Dans nos sociétés occidentales, au sein de l'espace européen, la France apparaît comme un des pays les moins tolérants dans le domaine de l'homoparentalité comparée aux Pays-Bas (pionnier en la matière), à la Suède, à l'Angleterre, à la Belgique... au Canada et à plusieurs Etats Nord-Américains Outre-Atlantique. Pour donner un exemple européen précis, en Espagne, en 1988, la Loi permet l'insémination artificielle aux couples lesbiens, plus tard, quatre régions autorisent l'adoption conjointe des couples gays et lesbiens. En 2005, la réforme du Code civil introduit l'entière égalité des droits pour le mariage et l'adoption, ce qui marque une étape historique et sociale importante dans un pays de tradition catholique.

\section{MISE EN QUESTIONS ET MISE EN DÉBAT}

Plusieurs éléments font nœuds dans le débat sur l'homoparentalité : la scène primitive et la question des origines, le désir d'enfant, la parenté biologique versus la parenté sociale, les repères sociaux qui concourent à la stabilité de la famille et à l'équilibre symbolique de notre société, et le développement des enfants qui grandissent dans des familles homoparentales.

\section{La scène primitive et la question des origines}

Qu'en est-il de la scène primitive, fondatrice de l'ordre familial symbolique? Winter [41] pose comme fondamental « le réel de la rencontre sexuelle aboutissant à la procréation » dans la structuration psychique de l'enfant, et affirme que l'enfant a besoin d'imaginer le coït parental (hétérosexuel) pour se construire. Dans le cas d'homoparents nous dit-il, "l'enfant va l'imaginer à partir de deux parents de même sexe et devra soutenir que l'impossible est possible ", même si les parents explicitent de façon claire à l'enfant comment il a été conçu. Cependant, lui-même atteste que l'enfant n'est pas seulement issu d'un seul rapport copulatoire et du fantasme de la scène primitive. Dans tous les cas et quelle que soit la configuration familiale, " il naît du rapport entre deux histoires familiales, deux généalogies, d'une rencontre d'imaginaires et de pensées ". C'est sans doute ici que l'enfant peut penser et répondre d'où il vient. Par ailleurs, nul besoin de rappeler que la scène primitive si 
elle n'est pas ancrée dans « la réalité » du coït parental peut être fantasmée, voire est toujours fantasmée. Ne l'est-elle pas déjà pour les couples hétérosexuels ayant recours à une Insémination avec donneur ? A partir d'entretiens menés auprès de couples lesbiens, Ducousso-Lacaze [14] nous montre aussi que " ces couples auraient à se représenter une scène primitive, celle qui a donné naissance à l'enfant, comportant nécessairement le donneur ".

Bien avant l'advenue des familles homoparentales et des problématiques et passions qu'elles soulèvent, des parentés sociales existaient déjà, déliées du rapport sexuel entre un homme et une femme, de la reproduction sexuée, bref de la procréation " ordinaire ". Par rapport au mythe fondateur, on ne s'appuie plus seulement sur la rencontre sexuelle et reproductive d'un homme et d'une femme. Ainsi en est-il de l'adoption des couples hétérosexuels pour lesquels n'existe aucun lien de sang entre les deux conjoints et l'enfant adopté1. "L'adoptant devient parent tout en étant complètement étranger à la « fabrication » génétique et biologique de l'enfant " [4]. Ainsi en est-il dans les familles recomposées pour lesquelles le "père social ", le " beau-père ", n'a aucun lien de sang avec les enfants de sa conjointe issus d'un précédent couple conjugal. Ainsi en est-il de l'insémination avec donneur pour laquelle il n'existe aucun lien biologique entre le père et l'enfant. " L'I.A.D. intervient pour reconstituer la chaîne de filiation et permettre que soit retrouvé le statut social de la paternité " [31]. Ces trois exemples illustrent bien que " ce n'est pas une contrainte naturelle qui noue le lien entre l'adulte et l'enfant mais une manifestation de la volonté " [4]. La reproduction sexuelle, la parenté biologique, sont ce faisant disjointes, déliées de la parenté sociale. Dans nos sociétés occidentales comme le souligne Godelier [18] " la parenté sociale est de retour ». C'est ainsi que le couple parental peut ne plus coïncider avec le couple conjugal (séparation conjugale, familles recomposées) ou encore que la parenté biologique ne coïncide pas forcément avec la parenté sociale (adoption, I.A.D...) $)^{2}$.

Cependant, dans tous les cas et quel que soit le système familial dans lequel il se situe, l'enfant a un « père » et une " mère " biologiques ou tout au moins leur patrimoine génétique. Quelle que soit la configuration, adoptive ou par insémination avec donneur, homosexuelle ou hétérosexuelle, la question posée reste identique et réfère aux origines : qui sont mes parents géniteurs dans le cas de l'adoption? d'où provient ce sperme et qui est mon géniteur dans la situation induite par une insémination avec donneur?

L'homoparentalité n'introduit pas de questions nouvelles à ce sujet, elle y fait écho et les rappelle. Le rapport originel doit-il être " naturel " ou " symbolique " ? De qui les enfants sont-ils nés ? Doivent-ils être le fruit d'un acte amoureux ou sont-ils le fruit de la rencontre de deux désirs ? Ces deux désirs doivent-ils être portés par un homme et une femme ou peuvent-ils être portés par deux hommes ou par deux femmes? La naissance puis le devenir de l'enfant s'inscrivent-ils dans l'altérité quand ses deux parents sont deux hommes ou deux femmes ou seule l'altérité des sexes parentaux est-elle nécessaire ? Ce faisant, ce père et cette mère sont-ils un homme et une femme ou seulement une position maternelle et paternelle ? Dans la Déclaration des droits de l'enfant du 20 novembre 1959, le mot « père » n'est jamais mentionné à la différence de ceux de " mère " et "parents ". Dans la Convention Internationale des Droits de l'enfant de 1989, plusieurs vocables apparaissent souvent associés entre eux. En dehors d'un article particulier qui concerne les soins pré et post-natals maternels, le mot " père " est toujours associé à celui de "mère ", le vocable " parents " associé à celui de "représentants légaux " (ou expressions assimilées). En 30 ans, les terminologies qui désignent les parents se sont donc modifiées mais à aucun moment n'apparaît l'appartenance sexuée, biologique de ceux qui sont nommés père, mère, parents.

Ainsi, les théories de la reproduction sexuée apparaissent insuffisantes à définir ce qu'est la filiation. "Pour faire de la parenté, il faut plus que deux organismes qui se reproduisent : il y a toujours un intervenant extérieur : un ancêtre ou un Dieu qui fait véritablement le lien entre l'enfant, ses parents et la société qui les reconnaît comme tel » [23]. La reproduction n'est pas la parenté ; la parenté n'est pas seulement une proximité de sang, elle s'inscrit dans un système plus large, moral, religieux, culturel et juridique. Ainsi, " ce n'est pas parce que nous maîtrisons aujourd'hui certains aspects de la reproduction que nous savons mieux ce qu'est un père, une mère, un fils, une fille. Quelles que soient les conditions dans lesquelles des cellules reproductrices sont utilisées, ce n'est pas l'origine biologique qui fonde la parenté. II faut toujours qu'une autorité morale, religieuse ou politique vienne le dire " [23]. Ainsi en est-il des adoptions, des inséminations avec donneur, et des situations homoparentales.

\section{Désir d'enfant}

Par le désir d'enfant, les couples homosexuels souhaitent accéder à la parentalité, s'inscrire dans l'ordre des générations et dans la filiation, faire de leurs parents des grandsparents dans le registre du don et de la dette, et renoncer ainsi à leur propre place d'enfant. Cependant, la relation homosexuelle n'est pas procréatrice, elle est stérile par essence. Certes, mais cette stérilité de fait n'a pas pour corollaire que le désir d'enfant n'existe pas au plan inconscient. Pour Flavigny [17], chez les homoparents existent à la fois un désir d'enfant conscient et une mise à distance de l'enfantement qui serait un désir inconscient opposé à l'enfant que témoigne leur homosexualité. Si cette opposition paradoxale désir d'enfant/impossible enfantement paraît pertinente, elle ne se limite pas aux couples homosexuels et peut être le support d'une réflexion dans d'autres situations. Par exemple, elle pourrait être discutée pour un couple hétérosexuel infertile pour lequel la vie sexuelle ne leur permet pas de procréer, de donner la vie au sein de leur couple alors qu'ils sont en âge de le faire. Qu'en est-il de leur désir d'enfant et à quelle place symbolique mettent-ils l'enfant dans leur demande d'accéder à la parentalité ? II y a bien ici aussi une dissociation de la sexualité, de la procréation et de l'accès à la parentalité. Autre exemple, l'impossible enfantement pourrait aussi être discuté chez les 
femmes hétérosexuelles, enceintes de multiples fois, et qui subissent des avortements itératifs dans un impossible enfantement.

Comme dans de nombreux couples, l'enfant est prolongement de soi-même, de valorisation narcissique, voire de remplacement... est-il pour autant l'objet « de toute puissance des adultes " ou de " complétude narcissique " voire un " objet partiel ». Là encore, ces propos, que l'on retrouve chez certains psychanalystes opposés à l'homoparentalité ou tout au moins soucieux de ce qu'elle peut entraîner/signifier au plan inconscient, ne sont pas réservés aux couples homosexuels qui désirent un enfant. Richard [35] à propos de la cure d'une patiente homoparentale relève à juste titre : " ces aléas me semblent le lot des couples, qu'ils soient hétérosexuels ou homosexuels, dont les membres présentent une structure de personnalité narcissique, i.e. dyadique, non triangulée ».

\section{Développement des enfants d'homoparents}

\section{a) Structuration psychique}

Les nouvelles structures familiales interrogent les théories psychanalytiques quand elles séparent la sexualité de la procréation ${ }^{3}$ et de la parenté. "Quels repères symboliques si chacun peut s'instituer parent à sa guise ? " interroge Meulders-Klein [32]. Quand les logiques sociales affrontent les logiques psychologiques, l'ordre symbolique est-il en contradiction avec l'ordre social ? Pour nombre de psychanalystes opposés à l'homoparentalité, le cadre symbolique relève du registre de l'inconscient et le cadre éducatif n'est qu'une facette du conscient qui servirait à masquer celle de l'inconscient. Dans la pratique clinique, sait-on comment se construisent les enfants d'homoparents au plan psychique, autour de l'CEdipe, du phallus, de la castration... ? Ces interrogations sont sans doute trop récentes pour que les réponses émises soient univoques, et c'est sans doute la raison pour laquelle elles suscitent des propos contradictoires parmi les psychanalystes. Plusieurs d'entre eux comme Winter, Eliacheff, Naouri mettent en exergue une mise en péril de la famille traditionnelle nucléaire de par la mise en péril de l'ordre symbolique fondé sur la différences des sexes et des générations, d'autres comme de Parseval, Tort, Roudinesco... émettent des éléments de réponse moins alarmistes et souvent plus nuancés.

Le principe du complexe d'CEdipe est fondé sur la triangulation et les psychanalystes soulignent la nécessité du tiers pour séparer l'enfant de sa mère (ou de celle/celui qui occupe cette position) afin de devenir un sujet sexué et séparé d'autrui. De fait, un des deux parents (quel que soit son sexe) peut donc prendre place de tiers et assurer la mise à distance de la fusion originelle. La fonction paternelle, et les psychanalystes le rappellent sans cesse, n'est pas une position mais une fonction qui peut être représentée par une autre personne que le père biologique, comme par exemple un grand-père, un oncle, un ami... L'essentiel est qu'elle s'exerce et qu'elle soit opérante. Mentionnons à cet endroit que pour tous les couples parentaux ordinaires hétérosexuels et homoparents, on peut être dans une position maternelle ou paternelle sans être garant d'une fonction symbolique maternelle et/ou paternelle, et que le tiers peut faire défaut, ce n'est pas l'apanage des homoparents de ne pas occuper ces fonctions et cette place de tiers. Cependant, la difficulté s'accroît si on considère comme Dor [13] que « le signifiant Nom du père doit être explicitement référé à l'existence d'un tiers marqué par la différence sexuelle par rapport au protagoniste qui se présente comme mère ". Sous cette hypothèse, la différence sexuelle apparaît centrale et pourrait donc ne plus être opérante dans des couples de même sexe. "Le besoin pour l'enfant d'avoir des référents des deux genres est reconnu par tous. Ce compagnon ou cette compagne ne joue donc pas, au moins au niveau explicite, le rôle de la mère ou du père non présent. II doit se placer ailleurs, on ne sait pas exactement où puisqu'aucun modèle de référence n'existe encore aujourd'hui » [12].

\section{b) Approche développementale}

Que sait-on du développement des enfants élevés par des homoparents? Une analyse critique des travaux empiriques disponibles sur le développement des enfants en familles homoparentales [40] a permis de montrer que, si ce type de recherches est encore rare (35 recherches recensées fin 2003), l'hégémonie américaine dans ce domaine est moins marquante qu'on ne pourrait le penser puisque près de la moitié de ces études sont européennes (surtout anglaises et belges). A propos de la rareté de telles recherches, Allen et Demo [1] rapportaient une certaine hésitation de la part des chercheurs à reconnaître le statut de famille aux couples homosexuels et leurs enfants. Notons aussi que la représentation des pères gays dans ces études est faible (un peu moins de $15 \%$ des recherches), sans doute parce qu'à l'inverse des lesbiennes ils disposent d'un accès restreint aux P.M.A. (mère porteuse) et peu obtiennent des agréments pour adopter. Les pères ont longtemps été (et le sont encore) pénalisés sur le plan de l'obtention de la garde des enfants qu'ils ont eus dans le cadre d'une union hétérosexuelle antérieure.

A propos des populations d'enfants élevés par des couples gays ou lesbiens, il apparaît globalement que les recherches ont davantage ciblé la tranche d'âge des enfants jeunes plutôt que celles des adolescents et des enfants devenus adultes, surtout depuis le début des années 1990. Cette réorientation paradigmatique est sans doute à mettre en lien avec le quasi abandon des thématiques centrées sur l'orientation sexuelle. Cette évolution notable s'accompagne également d'un affinement des âges ciblés : avant 1990 , les études portent plus souvent sur deux tranches d'âge $(50 \%)$, après 1990 , elles portent sur une seule tranche d'âge (58\%). Quant aux modes de filiation abordés, leur diversification est nette avec le temps : avant 1990 tous les enfants sont issus d'unions hétérosexuelles antérieures ; puis les situations homoparentales avec I.A.D. apparaissent en force dès le début des années 90 . Quant à la présence d'enfants adoptés, nés par mère porteuse ou par coparentalité, les travaux dans le domaine sont encore très rares. Cette diversité des situations prises en compte s'avère être un indicateur à la fois des progrès techniques dans le cadre des P.M.A., de l'évolution des familles et aussi de leur visibilité sociale. 
L'analyse des 35 recherches recensées a permis d'identifier une dizaine de thèmes dont l'aspect sexué/sexuel du développement des enfants, leurs problèmes psychologiques ou comportementaux, les relations avec les pairs et la stigmatisation, l'ajustement parents-enfants, la perception de soi, l'intelligence. D'autres thèmes sont traités de façon plus marginale : les sévices subis par les enfants, les contacts des enfants avec leurs grands-parents [24,39] et d'autres adultes hors du foyer, le concept de donneur chez les enfants nés par I.A.D. [36].

Concernant la question de la "transmission " de l'homosexualité des parents à leurs enfants, la douzaine de recherches fiables concernées ne fait pas état d'une présence disproportionnée d'une orientation homosexuelle chez les adolescent(e)s et adultes élevés ou ayant été élevés par des homosexuel(le)s seul(e)s ou en couple [5, 19, 21]. Une douzaine de recherches montre également que les enfants développent une identité sexuée conforme à leur sexe $[6,15,25]$. Sur le registre des problèmes émotionnels (anxiété, dépression...) et comportementaux (agressivité, délinquance...), les enfants n'apparaissent pas différents des enfants de familles hétéroparentales [38, 9, 33].

Sur le thème de la dynamique familiale, les résultats sont divers : la recherche de Golombok et al. [20] fait état d'interactions plus nombreuses dans les familles homoparentales que dans les autres, celle de Crosbie-Burnett et Helmbrecht [11] indique que la satisfaction familiale chez les enfants est meilleure quand ils considèrent le compagnon de leur pere biologique comme faisant partie de la famille. A ce propos, trois recherches montrent que la plupart des enfants étudiés incluent la compagne de leur mère biologique dans la famille $[6,15,37]$. Chez les enfants nés par I.A.D., les relations de l'enfant avec sa mère biologique et les relations avec la compagne de la mère biologique ne font pas l'objet d'une différence significative, notamment du point de vue de l'autorité et de l'acceptation [37, 38]. Par comparaison avec les pères des familles hétéroparentales, deux recherches montrent que les "belles-mères", dans les familles homoparentales, sont plus impliquées dans les activités avec les enfants que les pères des familles hétéroparentales [6, 37].

Une recherche sur le point de vue des enfants nés par insémination quant à la révélation ou le secret de leurs origines [36] indique que, sur une population de 41 enfants âgés de 7 à 17 ans, 54\% d'entre eux ne souhaitent pas connaître l'identité du donneur (et ce, sans différence significative selon le sexe des enfants), $27 \%$ souhaitent connaître son identité et $19 \%$ des enfants souhaitent uniquement des informations non-identifiantes. Enfin, $63 \%$ des enfants ne parlent jamais du donneur avec leurs mères. Ces résultats sont peut-être à mettre en lien avec le fait que la majorité des mères de ces enfants préfère que le donneur reste anonyme.

Enfin, les lieux de consultation en pédopsychiatrie ou en psychologie ne regorgent pas eux non plus d'enfants en souffrance psychique issus de foyers homoparentaux (si ce n'est de par la stigmatisation opérée sur leur famille et du silence que l'homosexualité des parents suppose). Rappelons s'il en est besoin, que les enfants de familles monoparentales minoritaires et exclus du système dominant des années 70 étaient accablés de tous les maux: anorexie, fugues, conduites addictives, problèmes scolaires... Trente ans plus tard, ces familles insérées dans notre système social et familial occidental ne sont plus stigmatisées de la même manière.

\section{PLURALITÉS DES FAMILLES HOMOPARENTALES}

En deux décennies la structure des familles homoparentales a évolué. Avant les années 90 , la majorité des enfants étaient issus d'union hétérosexuelle antérieure, à l'heure actuelle, le nombre d'enfants issus de l'adoption et des I.A.D. (y compris de " façon artisanale ") tend à augmenter (selon Clémençon \& Jourdan [10], la moitié des enfants seraient issus d'unions hétérosexuelles antérieures et l'autre moitié serait le fruit " d'un projet de coparentalité "). Les homoparentalités sont plurielles : union hétérosexuelle antérieure, adoption, mère "porteuse ", coparentalité, I.A.D... Cette évolution, sensible et rapide, est certainement due aux changements sociaux, aux coming-out (ce terme provient de l'expression "coming out the closet" qui signifie "sortie du placard" c'est-à-dire révéler son homosexualité à l'entourage familial, amical ou professionnel) au refus de la stigmatisation sociale (comme l'étaient en leur temps les familles monoparentales)... et aux progrès des sciences médicales.

De façon concomitante à cette évolution, on assiste de façon paradoxale, pour certaines familles homoparentales, à un retour vers la famille traditionnelle, le religieux, la morale via les demandes de mariages (pourquoi le mariage pour certains couples homosexuels - pas pour la majorité doit-il être une nécessité et doit-il être légalisé ?) et de baptêmes (en augmentation) ${ }^{4}$. Ces prises de positions sont d'ailleurs controversées par les gays et lesbiennes euxmêmes dans la mesure où elles s'attachent à revenir à un ordre moral et religieux. De même, dans les familles homoparentales, force est de constater que les stéréotypes traditionnels maternel versus paternel, masculin versus féminin restent prégnants et marqués, avec le souhait ainsi réalisé de se maintenir dans la norme maritale, sociale et culturelle. Ducousso-Lacaze [14] souligne aussi que là où on aurait pu s'attendre à de l'indifférenciation, « à la faveur de l'accès à la parentalité apparaissent de nouvelles différences entre les partenaires du couple, aussi bien en termes de rôles que de sentiment identitaire ". Comme le repère Mailfert [30] il existe, pour certains homoparents, une volonté de s'intégrer dans l'ordre social, de s'inscrire dans un système familial, dans la normalité familiale, aspiration que l'on retrouve au travers des actes de la vie quotidienne, mais aussi dans les appellations de parenté (" maman ", " marraine "...) qui " traduisent une volonté de consolider les rôles et les statuts" traditionnellement définis. Même si les conduites ne se différencient pas au plan domestique, cela « n'implique pas une indifférenciation des conduites vis-àvis de l'enfant » [12], l'un peut jouer davantage le rôle d'autorité, c'est souvent celui qui est considéré comme le « vrai parent» - en référence à l'histoire de la naissance, et à la qualité « biologique » de son implication. 


\section{EN GUISE DE CONCLUSION INTERROGATIVE ET RÉFLEXIVE}

Qu'est-ce qui fait la famille ? Qu'est-ce qui la fonde ? Les évolutions sociales, culturelles, morales et juridiques du système familial entraînent-elles une remise en question des modèles théoriques traditionnels, notamment ceux issus de la psychanalyse ? Comment penser la procréation, la parenté face aux progrès de la science, quand elle se déconnecte de la sexualité ? La " parenté plurielle " [7], la pluriparentalité $[16,28]$ et parmi elle l'homoparentalité $[3$, 22]) nous amènent à une remise en question des conceptions traditionnelles de la sexualité, de la procréation (et de la contraception), de la famille structurée sur l'altérité sexuelle et sexuée des parents et sur la filiation biologique. La théorie psychanalytique s'applique à un modèle familial nucléaire, biparental. Est-elle de ce fait applicable à d'autres modèles familiaux, à d'autres positions et fonctions parentales ? Doit-on repenser ce modèle eu égard aux changements (toute psychologie est une psychologie sociale nous disait Freud) ? Ou bien, doit-on élaborer une théorie qui s'appliquerait à d'autres modèles parentaux ? " La clinique psychanalytique se doit, elle aussi, de s'intéresser à ces destins, car ils témoignent de certains de ces oripeaux dont Narcisse et CEdipe se pareront durant le troisième millénaire " [35].

L'homoparentalité suscite de multiples réflexions et instaure des débats passionnés voire polémiques, dans la mesure où elle fait partie dorénavant de notre paysage social (en France, l'homoparentalité concerne environ $11 \%$ de couples lesbiens et $7 \%$ de couples gays ; soit une centaine de milliers de foyers en situation homoparentale selon l'APGL). Pour celles et ceux qui craignent le désordre sociétal, nous nous appuierons sur les propos de Godelier [18] : " si le système familial bouge, les liens sociaux ne s'effondrent pas pour autant. II existe une confusion entre les liens familiaux, de parenté et sociétal. Ils en font partie mais ne constituent pas la société ".

Pour que le débat puisse se poursuivre, rappelons pour mémoire, ce qu'énonçait l'Académie française des Sciences Morales et Politiques condamnant fermement I'I.A.D. : " c'est une atteinte aux assises du mariage, de la famille et de la société par le fait d'intégrer frauduleusement dans une famille, un enfant qui porte le nom du père légal et qui s'en croira le fils ". Au vu de l'évolution de cette technique médicale au fil du temps et en raison de l'intérêt pour les familles qui l'utilisent, ne participons pas à la « condamnation " des familles homoparentales et des enfants qui grandissent parmi elles. En tant que chercheurs, nous n'avons ni à émettre de jugement de valeur ni à nous référer à la morale mais nous avons pour mission de dégager des interrogations pertinentes eu égard à notre champ disciplinaire de référence, et de proposer quelques éléments de réponse même s'ils ne sont que fragmentaires, hétérogènes voire pour l'heure insatisfaisants, et susceptibles d'être à leur tour remis en question.

\section{RÉFÉRENCES}

1. ALLEN K.R., DEMO D.H. : The families of lesbians and gay men : a new frontier in family research. Journal of Marriage and the Family, 1995, $57: 111-127$.

2. APGL : Familles gayes et lesbiennes en Europe. In : Gross M. ed. Homoparentalités, Etats des lieux, Issy-les-Moulineaux, ESF, 1999.

3. BEAUMATIN A., RICAUD-DROISY H., ESPIAU G. : Approche psychologique de l'exercice de la parentalité chez les couples homosexuels. Pratiques Psychologiques, 2003, 2 : 45-54.

4. BORILLO D., PITOIS-ETIENNE T., 2003 : http://www.psydesir.com

5. BOZETT F. : Social control of identity by children of gay fathers. Western Journal of Nursing Research, 1988, $10: 550-565$.

6. BREWAEYS A., VAN HALL E.V. : Lesbian motherhood : the impact on child development and family functioning, $J$ Psychosom. Obst. Gynaecol., 1997, 18 : 1-16.

7. CADORET A. : Parenté plurielle. Paris, L'Harmattan, 1995.

8. CHAN R.W., BROOKS R.C., RABOY B., PATTERSON C.J. : Division labor among lesbian and heterosexual parents : associations with children's adjustment. Journal of Family Psychology, 1998, 12 : 402-419.

9. CHAN R.W., RABOY B., PATTERSON C.J. : Psychosocial adjustment among children conceived by lesbian and heterosexual mothers. Child Dev., 1998, 69 : 443-457.

10. CLEMENCON L., JOURDAN V., 2004 : http://homoparentalite.free.fr/PCE.2.doc

11. CROSBIE-BURNETT M., HELMBRECHT L. : A descriptive empirical study of gay male stepfamilies. Family Relations, $199342: 256-262$

12. De SINGLY F., DESCOUTURES V., 1999 : http://www.france.qrd.org/assocs/apgl/documents/f_singly1.htm

13. DOR J. : Structure et perversions. Paris, Denoël, 1987.

14. DUCOUSSO-LACAZE A. : Familles homoparentales : qu'estce qui (ne) change (pas) ? Psychologie et Média, 2005, 2 : 3840 .

15. DUNDAS S., KAUFMAN M. : The Toronto Family Study. Journal of Homosexuality, 2000, $40: 65-79$.

16. FINE A. : Vers une reconnaissance de la pluriparentalité. Esprit, 2001, mars/avril : 40-53.

17. FLAVIGNY C. : Le désir d'enfant des homosexuel(le)s. Journal des Psychologues, 2002,195 : 26-28.

18. GODELIER M. : Les métamorphoses de la parenté. Paris, Fayard, 2004.

19. GOLOMBOK S., TASKER F. : Do parents influence the sexual orientation of their children ? Findings from a longitudinal study of lesbian families. Dev. Psychol., 1996, 32 : 3-11.

20. GOLOMBOK S., TASKER F., MURRAY C. : Children raised in fatherless families from infancy : family relationships and socioemotional development of children of lesbian and heterosexual mothers. J. Child Psychol. Psychiatry, 1997, 38 : 783791.

21. GOTTMAN J. : Children of gay and lesbian parents. In : Bozett F., Sussman M. eds. Homosexuality and family relations. New York, Haworth Press, 1990 : 177-196. 
22. GROSS M. : Homoparentalités, état des lieux. Ramonville Saint-Agne, Erès, 2005.

23. JOURNET N. : Les cinq leçons de Maurice Godelier. Sciences Humaines, 2004, $156: 34-39$.

24. JULIEN D., BUREAU M.F., LEBLOND DE BRUMATH A. : Grand-parentalité et homoparentalité au Québec. Nouvelles dispositions législatives et proximité des liens en fonction de la composition familiale. In : Schneider B., Mietkiewicz M-C, Bouyer S. eds. Grands-parents et grand-parentalité. Ramonville Saint-Agne, Erès, 2005 : 199-217.

25. KIRKPATRICK M., SMITH C., ROY R. : Lesbian mothers and their children : a comparative survey. American Journal of Orthopsychiatry, 1981, $51: 545-551$.

26. LAMB M.E., PLECK J.H., CHARNOV E.L., LEVINE L.A. : A biosocial perspective on paternal behavior and involvement. In : Lancaster J.B., Altam J., Rossi A.S., Sherrod L.R. eds. Parenting across the lifespan : Biosocial dimensions. NewYork, Aldine de Gruyter, 1987 : 111-142.

27. LE CAMUS J., ZAOUCHE-GAUDRON C. : La présence du père auprès du jeune enfant : de l'implication accrue à l'implication congrue. Psychiatrie de l'Enfant, 1998, XLI : 297-319.

28. LE GALL D., BETTAHAR Y. : La pluriparentalité. Paris, PUF, 2001.

29. LEROY-FORGEOT F. : Les enfants du Pacs : réalités de I'homoparentalité. Paris, L'atelier de l'Archer, 1999.

30. MAILFERT M., 2004 : http://revel.unice.fr/anthropo

31. MÉTRO N., GAUTHIER P. : La paternité par Insémination Artificielle avec Donneur. In : Fine A., Laterrasse C., ZaoucheGaudron C. eds. A chacun sa famille. Toulouse, Editions Universitaires du Sud, $1998: 65-79$.

32. MEULDERS-KLEIN M.T. : La place du père dans l'ordre symbolique des lois. L'un et l'autre ? Ou l'un est l'autre ? La pensée, 2001, $327: 31-46$

33. NADAUD S. : Approche psychologique et comportementale des enfants vivant en milieu homoparental. Thèse de médecine, Université Bordeaux 2, 2000.

34. PAQUETTE D. : La relation père-enfant et l'ouverture au monde. Enfance, 2004, 2 : 205-225.

35. RICHARD H. : Désir d'enfant ? la maternité planifiée chez les couples lesbiens: un début de réflexion clinique. Psychothérapies, 2000, 20, : 115-124.

36. VANFRAUSSEN K., PONJAERT-KRISTOFFERSEN I., BREWAEYS A. : An attempt to reconstruct children's donor concept : a comparison between children's and lesbian parents' attitudes towards donor anonymity. Hum. Reprod., 2001, $16: 2019-2025$

37. VANFRAUSSEN K., PONJAERT-KRISTOFFERSEN I., BREWAEYS A. : Family functioning in lesbian families created by donor insemination. American Journal of Orthopsychiatry, $2003,73: 78-90$

38. VANFRAUSSEN K., PONJAERT-KRISTOFFERSEN I., BREWAEYSA. : Why do children want to know more about the donor? The experience of youngsters raised in lesbian families. J. Psychosom. Obst. Gynaecol., 2003, 24 : 31-38.

39. VECHO O., SCHNEIDER B., ZAOUCHE-GAUDRON C. : Grand-homoparentality : Gay and lesbian couples representation. Xth International Congress on Family Education Family's
Strong and Weak Points in Changing Societies, Las Palmas de Gran Canaria (Espana), 16th - 19th March 2005.

40. VECHO O., SCHNEIDER B. : Homoparentalité et développement de l'enfant : bilan de 30 ans de publications. Psychiatrie de l'enfant, 2005 (à paraître).

41. WINTER J.P., 2003 : http://homoparentalite.free.fr/etudes/nouvelobs.htm

42. ZAOUCHE-GAUDRON C. : La différenciation paternelle et le père suffisamment présent. Neuropsychiatrie de l'enfance et de l'adolescence, 1997, $45: 101-105$.

43. ZAOUCHE-GAUDRON C. : La problématique paternelle. Ramonville Saint-Agne, Erès, 2001.

Communication au XII ${ }^{\circ}$ Séminaire de la Fédération Française des CECOS, Tours, 7-9 Avril 2005.

Manuscrit reçu : mars 2005 ; accepté mai 2005.

\section{Notes}

1 Relevons le cas particulier de l'adoption en France (notamment dans le cas des familles monoparentales) et le mensonge hypocrite qu'induit notre société de taire l'homosexualité pour que des hommes et des femmes puissent adopter des enfants.

2 La parenté sociale disjointe de la parenté biologique n'est pas un fait nouveau en anthropologie. Dans certaines sociétés, le couple conjugal n'est pas le couple parental: on peut par exemple repérer dans certains pays d'Afrique que l'oncle maternel fait figure de père social ou encore chez les $\mathrm{Na}$ de Chine que le père géniteur est inconnu, et chez les Samos que l'enfant n'est pas de l'époux légitime.

3 N'oublions pas le clivage déjà réalisé dès lors que les méthodes contraceptives ont été introduites, séparant elles aussi sexualité et procération.

4 Rappelons avec Godelier [18] que c'est l'église qui, à partir du Vème siècle, a imposé les règles de la famille toujours à l'œuvre à l'heure actuelle et qu'elle continue à " réprouver les P.M.A. parce qu'elles ne respectent pas les modes nature/s de conception en dissociant vie sexuelle et procréation " [31].

\section{Remerciements :}

Nous tenons à remercier Gérard Neyrand, Sociologue, et Patrick Ben Soussan, Pédopsychiatre, pour leur lecture attentive, leurs remarques judicieuses et leurs précieux conseils. 
Questions concerning same-sex parenthood

Chantal ZAOUCHE-GAUDRON, Olivier VECHO

Same-sex parenthood is the subject of often heated debate, as these new family structures separate sexuality from procreation and parenthood. Following a brief review of the history of same-sex parenthood, emphasizing the changes in the paradigms of psychology and taking into account data derived from anthropology, the authors examine the main questions raised by the appearance of same-sex families.

The debate on same-sex parenthood focuses on several elements, particularly with reference to psychoanalytical theories: the primal scene and the question of origins, the desire for children, and the psychic structuring of the child. These various points will be examined and discussed. The development of children who grow up in samesex families will be examined on the basis of the data of the scientific literature. Analysis of $\mathbf{3 5}$ studies identified ten main themes including the sexed/sexual aspect of child development, their psychological or behavioural problems, relations with their peers and stigmatisation, parent-child adjustment, etc. Lastly, the authors discuss the rapid inscription of same-sex parenthood in our social landscape and, concomitantly with this development, the paradoxical adhesion of some same-sex couples to the norms of the traditional family.

Key words : Same-sex parenthood, primal scene, secret of origins, desire for children, child development 